\title{
Increased diversity space-time coding using the diversity transform
}

\author{
Amir J Salomon* and Ofer Amrani*
}

\begin{abstract}
The paper presents a method for constructing space-time block codes for multiple-input multiple-output channels by concatenating orthogonal designs with the so-called diversity transform. Relying on unitary transforms, the diversity transform increases the channel alphabet without sacrificing information rate, bandwidth, or Euclidean distance. The distribution of the resulting channel alphabet is shown to quickly become Gaussian-like. Specific code matrices are constructed and optimized based on the cutoff rate. Both optimum and, reduced-complexity, suboptimum detection algorithms are presented. Simulation results are provided for demonstrating the gains attainable when using the proposed codes.
\end{abstract}

Keywords: Space-time coding; Error correcting codes; Diversity; Wireless channels

\section{Introduction}

Wireless channels often suffer from severe signal attenuation due to multipath propagation. Consequently, the receiver faces greater difficulty in determining the transmitted signal reliably unless some less attenuated replica of the signal is somehow available. This serves as the basis for an approach, generally known as diversity, which is achievable via temporal, frequency, polarization, or spatial resources. In some applications, practical means of achieving diversity is by deploying multi-element antenna arrays at the transmitter and/or receiver side.

Alamouti [1] presented an effective communication scheme employing two transmit antennas jointly with a simple detection procedure. Space-time (ST) block codes, introduced by Tarokh et al. [2], generalize Alamouti's scheme for an arbitrary number of transmit antennas. These codes exploit the theory of orthogonal designs and achieve the full diversity attainable with any configuration of transmit and receive antennas.

Providing time diversity via classical coding techniques typically entails frequency expansion. There are various solutions to this problem - most relevant to the current work include high diversity lattices, see, e.g., $[3,4]$ and the references therein, and the so-called diversity transform (DRT) [5,6]. The diversity transform increases the

*Correspondence: amirj@eng.tau.ac.il ; ofera@eng.tau.ac.il Department of Electrical Engineering-Systems Ramat Aviv, Tel Aviv University, Tel-Aviv 69978, Israel channel alphabet, and hence the diversity, by employing appropriate unitary matrix multiplication.

The code construction presented herein is aimed at combining the properties of the two aforementioned approaches in a manner that facilitates computationally efficient encoding and detection. The resulting codes may be considered as multi-layered space-time codes, whereby coding based on orthogonal designs is applied to each layer, and where the different layers are linearly dependent via some unitary transformation. This multi-layered structure improves the diversity without sacrificing bandwidth, while producing channel alphabet whose distribution quickly becomes Gaussian-like (the distribution appears as sampled Gaussian function with a number of samples proportional to the dimension of the DRT and the constellation used).

For notational brevity, we shall henceforth refer to the obtained serially concatenated codes as diversified spacetime (DST) codes.

DST codes provide high degree of flexibility in adapting the code parameters to any antenna configuration and channel coherence time, which, in turn, determines the size of the diversity transform to be employed. A DST code can be very short, as short as two code words of the constituent ST code. Thus, it may introduce very small detection delay, while still providing high performance gain, compared to the constituent ST codes, with low

\section{Springer}

(c) 2014 Salomon and Amrani; licensee Springer. This is an Open Access article distributed under the terms of the Creative Commons Attribution License (http://creativecommons.org/licenses/by/2.0), which permits unrestricted use, distribution, and reproduction in any medium, provided the original work is properly cited. 
encoding and detection complexity. In general, implementation complexity is only slightly higher than that of the constituent ST code. This is compared to constructions which have promising information theoretic properties [7], but their detection is typically hard to realize.

A different interesting approach for serially concatenating ST codes with rate-1 codes was introduced before. Therein, Lin and Blum [8] employed a rate 1 recursive code, with feedback polynomial $(1+D)$. ST coding is used as the outer code, and so the obtained alphabet consists of the symbols of the constellation used, which is typically quadrature amplitude modulation (QAM). This concatenation approach along with the suggested iterative detection scheme [8] is mentioned to be more suitable for applications which can tolerate some delay.

While coding schemes that use rotation transformation within a codeword are known, see, e.g., the seminal work of Boutros and Viterbo [9], herein, we attempt to construct and optimize a transform that operates concurrently on a plurality of codewords of a known scheme with minimum added detection complexity.

The rest of the paper is organized as follows. Background material and definitions are given in Section 2. The proposed DST code construction is presented in Section 3 along with analysis of the code's alphabet distribution. DRT matrices are constructed and optimized based on the cutoff rate in Section 4. The optimization is carried out assuming that the average received SNR is known to the transmitter while channel state information (CSI) is required only at the receiver side. In Section 5, a two-stage suboptimum detection algorithm is presented based on linear estimation. Optimum detection is also briefly mentioned. Section 6 provides simulation results for a rich set of scenarios and DST codes of bandwidth efficiencies 1,2 , and $3 \mathrm{bits} / \mathrm{s} / \mathrm{Hz}$. Finally, conclusions are drawn in Section 7.

\section{Background}

\subsection{The channel model}

We consider a wireless communication system where the transmitter is equipped with $n$ antennas and the receiver with $m$ antennas. At each time slot $t$, complex symbols $y_{i}^{t}$, $i=1,2, \ldots, n$, are transmitted simultaneously from the $n$ transmit antennas. We denote by $h_{i, j}^{t}$ the path gain from transmit antenna $i$ to receive antenna $j$ at time slot $t$. The different paths are assumed frequency-nonselective, and their gains are modeled as samples of independent complex Gaussian random variables with variance $\frac{1}{2}$ per real dimension. The channel is of block-fading type, meaning that the path gains $h_{i, j}^{t}$ are constant over several time slots to which we refer as a block. The path gains are assumed statistically independent among different blocks. A single DST codeword, or code array as it will be called in the sequel, spans over several channel realizations.
At time $t$, the value $r_{j}^{t}$, received at antenna $j$, is given by:

$$
r_{j}^{t}=\sum_{i=1}^{n} h_{i, j}^{t} y_{i}^{t}+\eta_{j}^{t}
$$

where the noise samples $\eta_{j}^{t}$ are independent samples of a zero-mean complex Gaussian random variable with variance $n /(2 \mathrm{SNR}) \equiv \sigma^{2} / 2$ per real dimension. The average energy of the symbols transmitted from each antenna is normalized to be one, so that the average power of the received signal at each receive antenna is $n$ and the signal-to-noise ratio is SNR.

\subsection{Codes from orthogonal designs}

\subsubsection{Code construction}

Space-time block codes constructed from orthogonal designs were introduced by Tarokh et al. [2] as a generalization of the so-called Alamouti scheme [1]. A space-time code is defined as a $p \times n$ transmission matrix $G$ with orthogonal columns. The entries of the matrix $G$ are linear combinations of the complex variables $x_{1}, x_{2}, \ldots, x_{k}$ and their conjugates; these variables are actually the source (data) symbols to be transmitted. Since $p$ time slots are allocated for transmitting $k$ symbols, the symbol rate of the code is $R=k / p$.

For example, $G_{2}$ is the Alamouti code for two transmit antennas; it has rate $R=1$ (as $p=k=n=2$ ):

$$
G_{2}=\left(\begin{array}{rr}
x_{1} & x_{2} \\
-x_{2}^{*} & x_{1}^{*}
\end{array}\right) .
$$

Other codes that will be used in this work are the rate half codes, $G_{3}$ and $G_{4}$, and rate $3 / 4$ codes $H_{3}$ and $H_{4}$, given in Appendix.

\subsubsection{Conventional detection scheme}

For completeness, we briefly review the detection process used for the abovementioned ST codes. The symbols $x_{1}, x_{2}, \ldots, x_{k}$ can be detected at the receiver via linear processing. As an example, for the code $G_{2}$, the receiver constructs the next two values:

$$
\begin{aligned}
& \tilde{x}_{1}=\sum_{j=1}^{m}\left(h_{1, j}^{*} r_{j}^{1}+h_{2, j}\left(r_{j}^{2}\right)^{*}\right), \\
& \tilde{x}_{2}=\sum_{j=1}^{m}\left(h_{2, j}^{*} r_{j}^{1}-h_{1, j}\left(r_{j}^{2}\right)^{*}\right) .
\end{aligned}
$$

Plugging Equation 1 into Equation 3, it is easy to derive: $\tilde{x}_{1}=\tilde{h} \cdot x_{1}+\tilde{\eta}_{1}$; and $\tilde{x}_{2}=\tilde{h} \cdot x_{2}+\tilde{\eta}_{2}$, where $\tilde{h}=$ $\sum_{j=1}^{m}\left(\left|h_{1, j}\right|^{2}+\left|h_{2, j}\right|^{2}\right)$ and $\tilde{\eta}_{1}, \tilde{\eta}_{2}$ are independent, zero mean, complex Gaussian random variables (given $h_{i, j}$ at the receiver) with variance $\frac{\sigma^{2}}{2} \cdot \tilde{h}$ per real dimension. This implies that optimal reconstruction of $x_{1}$ and $x_{2}$ is possible from $\tilde{x}_{1}$ and $\tilde{x}_{2}$, respectively. Generalizing this: 
Proposition 1. For any space-time block code constructed from orthogonal design with variables $x_{1}, x_{2}, \ldots, x_{k}$, and assuming perfect knowledge of channel path gains at the receiver, one can construct any $\tilde{x}_{l, 1 \leq l \leq k}$ using linear processing:

$$
\tilde{x}_{l}=\tilde{h} \cdot x_{l}+\tilde{\eta}_{l}
$$

where $\tilde{h}=\alpha \cdot \sum_{i=1}^{n} \sum_{j=1}^{m}\left|h_{i, j}\right|^{2}$, $\alpha$ is a constant (which depends on the specific orthogonal code used), and $\tilde{\eta}_{l, 1 \leq l \leq k}$ are independent zero mean complex Gaussian random variables with variance $\frac{\sigma^{2}}{2} \cdot \tilde{h}$ per real dimension.

Henceforth, without loss of generality, it is assumed that $\alpha=1$, since one can divide both sides of Equation 4 by $\alpha$ and obtain the same model (up to the noise variance).

\section{Diversified space-time codes}

Let $Y \in C^{N}$ denote an $N$-dimensional vector whose entries belong to a complex alphabet of cardinality $M$. The diversity transform is a linear operation on $Y$ represented as

$$
X=\mathcal{A} Y
$$

where $\mathcal{A}$ is an $N \times N$ unitary matrix. This transform increases diversity owing to increased alphabet size [5,10]; it preserves the average energy and the minimum Euclidean distance of the input set $\{Y\}$. In Subsection 3.3, we show that the output set $\{X\}$ quickly becomes Gaussian-like (with the increase of $N$ ).

\subsection{General coding description}

The diversity transform and orthogonal designs, described in Section 2.2, can be combined so as to provide a coding scheme of increased diversity. The proposed scheme employs an $\mathrm{N}$-dimensional diversity transform combined with $N$ space-time codewords, each of rate $R=\frac{k}{p}$.

Let $x_{i}^{j}, 1 \leq j \leq N, 1 \leq i \leq k$, denote the symbol $x_{i}$ to be transmitted by the $j$ th ST codeword. Correspondingly, define $k$ vectors of the form

$$
X_{i}=\left[x_{i}^{1} x_{i}^{2} \ldots x_{i}^{N}\right]^{T},
$$

where each of these vectors is obtained by multiplying a (source) $N$-dimensional vector $Y_{i}$ by the DRT matrix $\mathcal{A}$ (5). The second step is to encode the $N k$-dimensional vectors $\left[x_{1}^{j} x_{2}^{j} \ldots x_{k}^{j}\right], 1 \leq j \leq N$, into $N$ ST codewords, which constitute a single DST code array.

The proposed coding scheme is best explained by means of an example using $N=2$. In this case, we use DRT of order 2 and two transmit antennas. Denote by $Y_{1}=$ $\left[\begin{array}{ll}y_{1}^{1} & y_{1}^{2}\end{array}\right]^{T}, Y_{2}=\left[\begin{array}{ll}y_{2}^{1} & y_{2}^{2}\end{array}\right]^{T}$ a set of symbols from a given constellation. First, use the diversity transform matrix $\mathcal{A}$ to obtain two so-called diversified vectors

$$
\begin{aligned}
& X_{1}=\left[\begin{array}{l}
x_{1}^{1} \\
x_{1}^{2}
\end{array}\right]=\mathcal{A}\left[\begin{array}{l}
y_{1}^{1} \\
y_{1}^{2}
\end{array}\right]=\left[\begin{array}{l}
a_{11} y_{1}^{1}+a_{12} y_{1}^{2} \\
a_{21} y_{1}^{1}+a_{22} y_{1}^{2}
\end{array}\right] ; \\
& X_{2}=\left[\begin{array}{l}
x_{2}^{1} \\
x_{2}^{2}
\end{array}\right]=\mathcal{A}\left[\begin{array}{l}
y_{2}^{1} \\
y_{2}^{2}
\end{array}\right]=\left[\begin{array}{l}
a_{11} y_{2}^{1}+a_{12} y_{2}^{2} \\
a_{21} y_{2}^{1}+a_{22} y_{2}^{2}
\end{array}\right] .
\end{aligned}
$$

Next, two ST codewords are generated:

first ST codeword

$$
\left(\begin{array}{cc}
x_{1}^{1} & x_{2}^{1} \\
-x_{2}^{1 *} & x_{1}^{1 *}
\end{array}\right)=\left(\begin{array}{cc}
a_{11} y_{1}^{1}+a_{12} y_{1}^{2} & a_{11} y_{2}^{1}+a_{12} y_{2}^{2} \\
-\left(a_{11} y_{2}^{1}+a_{12} y_{2}^{2}\right)^{*} & \left(a_{11} y_{1}^{1}+a_{12} y_{1}^{2}\right)^{*}
\end{array}\right)
$$

and a second ST codeword

$$
\left(\begin{array}{cc}
x_{1}^{2} & x_{2}^{2} \\
-x_{2}^{2 *} & x_{1}^{2 *}
\end{array}\right)=\left(\begin{array}{cc}
a_{21} y_{1}^{1}+a_{22} y_{1}^{2} & a_{21} y_{2}^{1}+a_{22} y_{2}^{2} \\
-\left(a_{21} y_{2}^{1}+a_{22} y_{2}^{2}\right)^{*} & \left(a_{21} y_{1}^{1}+a_{22} y_{1}^{2}\right)^{*}
\end{array}\right) .
$$

The two ST component codewords, which constitute a single DST code array, are transmitted one following the other.

Stated more generally, any orthogonal design code can be represented as a $p \times n$ matrix, $n$ being the number of transmit antennas:

$$
\begin{aligned}
G\left(x_{1}, \ldots, x_{k}\right) \\
\quad=\left(\begin{array}{cccc}
g_{11}\left(x_{1}, \ldots, x_{k}\right) & g_{12}\left(x_{1}, \ldots, x_{k}\right) & \ldots & g_{1 n}\left(x_{1}, \ldots, x_{k}\right) \\
g_{21}\left(x_{1}, \ldots, x_{k}\right) & g_{22}\left(x_{1}, \ldots, x_{k}\right) \ldots & g_{2 n}\left(x_{1}, \ldots, x_{k}\right) \\
\vdots & \vdots & \vdots & \vdots \\
g_{p 1}\left(x_{1}, \ldots, x_{k}\right) & g_{p 2}\left(x_{1}, \ldots, x_{k}\right) & \ldots & g_{p n}\left(x_{1}, \ldots, x_{k}\right)
\end{array}\right)
\end{aligned}
$$

where $g_{i j}\left(x_{1}, \ldots, x_{k}\right)$ is a (complex) function of $k$ variables. Now, we take a group of $k$ vectors $Y_{i}, 1 \leq i \leq k$, each vector $Y_{i}$ consists of $N$ symbols, which represent the source information. Using these vectors, we create a group of $k$ vectors $X_{i}$ (each of length $N$ ), created using the diversity transform:

$$
X_{i}=\mathcal{A} Y_{i},
$$

The elements of each vector $X_{i}$ are denoted by $X_{i}=$ $\left[x_{i}^{1} x_{i}^{2} \ldots x_{i}^{N}\right]^{T}$.

A DST code array can thus be simply described as a sequence of $N$ ST component codewords in the form:

$$
\left[\begin{array}{c}
G\left(x_{1}^{1}, x_{2}^{1}, \ldots, x_{k}^{1}\right) \\
G\left(x_{1}^{2}, x_{2}^{2}, \ldots, x_{k}^{2}\right) \\
\vdots \\
G\left(x_{1}^{N}, x_{2}^{N}, \ldots, x_{k}^{N}\right)
\end{array}\right] .
$$


Symbols from consecutive ST component codewords are interrelated via the diversity matrix $\mathcal{A}$. Note that in practice, these ST component codewords do not have to be transmitted consecutively in time. Rather, some form of interleaving can be applied in order to maximize the diversity of the DST code array for the price of somewhat increased decoding latency. A DST code array conveys a total of $k \cdot N$ source symbols using $p \cdot N$ channel uses, while the component ST orthogonal design code conveys $k$ source symbols using $p$ channel uses, i.e, coding rate of $k / p$ symbols per channel use is preserved.

\subsection{Receiver-side description}

At the receiver side, the ST codewords are decoded first by employing linear processing in accordance with Proposition 1 . Thus, $k N$-dimensional vectors $R_{i}, 1 \leq i \leq k$, are obtained each of which is related to $X_{i}$ and $Y_{i}$ by ${ }^{\mathrm{a}}$

$$
R_{i}=H X_{i}+\Gamma_{i}=H \mathcal{A} Y_{i}+\Gamma_{i}
$$

where $H$ is an $N \times N$ diagonal matrix given by:

$$
H=\left[\begin{array}{c}
\tilde{h}^{1}, 0,0, \ldots, 0 \\
0, \tilde{h}^{2}, 0, \ldots, 0 \\
0, \ldots, \tilde{h}^{i}, \ldots, 0 \\
\vdots \\
0,0, \ldots, 0, \tilde{h}^{N}
\end{array}\right],
$$

with $\tilde{h}^{t}=\sum_{i=1}^{n} \sum_{j=1}^{m}\left|h_{i, j}^{t}\right|^{2}, h_{i, j}^{t}$ being the path gain from transmit antenna $i$ to receive antenna $j$ for ST codeword $t$, and where $\Gamma_{i}$ is an $N$ dimensional vector of independent complex Gaussian random variables, $\Gamma_{i}=$ $\left[\tilde{\eta}_{i}^{1}, \tilde{\eta}_{i}^{2}, \ldots, \tilde{\eta}_{i}^{N}\right]^{T}$, with $E\left\{\Gamma_{i} \Gamma_{i}^{\dagger}\right\}=\sigma^{2} \cdot H$. Detailed description of the detection process is deferred to Section 5 .

\subsection{DST codes - alphabet distribution}

The DST code construction presented in this section, and particularly the use of high-diversity transforms, results with code alphabet of increased cardinality and nonuniform distribution which tends to approach (discrete) Gaussian even for small transform dimensions.

Figure 1 demonstrates the last assertion. The statistical distribution of the elements of $X$ is plotted: $Y$ is chosen from BPSK constellation, and normalized Hadamard matrices of increasing order are used as representing the transform matrix $\mathcal{A}$. The convergence to Gaussian distribution is evident as the transform order, $N$, is increased.

Taking $N$ to be asymptotically large, the following property of the DST construction is simple to prove.

Proposition 2. The DST code output $X$ approaches a Gaussian vector as $N \rightarrow \infty$.
Proof. This proposition follows simply from the central limit theorem.

Proposition 3. If $\mathcal{A}$ is an orthonormal matrix and the components of $Y$ are uniformly, symmetrically distributed around $O$ and obeying the power constraint $E\left(y_{i}^{2}\right)=P$, then in the limit $N \rightarrow \infty$, the components of $X$ are statistically independent, Gaussian distributed with zero mean and variance $P$.

Proof. $E\left(y_{i}\right)=0$ due to the symmetrical distribution of $Y$ around 0; therefore:

$$
E\left(x_{i}\right)=E\left(a_{i, 1} y_{1}+a_{i, 2} y_{2}+\ldots+a_{i, N} y_{N}\right)=0,
$$

and

$$
\begin{aligned}
E\left(x_{i} x_{j}\right)= & E\left[( a _ { i , 1 } y _ { 1 } + a _ { i , 2 } y _ { 2 } + \ldots + a _ { i , N } y _ { N } ) \left(a_{j, 1} y_{1}+a_{j, 2} y_{2}\right.\right. \\
& \left.\left.+\ldots+a_{j, N} y_{N}\right)\right]=a_{i, 1} a_{j, 1} E\left(y_{1}^{2}\right)+a_{i, 2} a_{j, 2} E\left(y_{2}^{2}\right)+\ldots \\
& +a_{i, N} a_{j, N} E\left(y_{N}^{2}\right)=P \cdot \delta(i, j),
\end{aligned}
$$

where $\delta(i, j)=1$ for $i=j$ and 0 otherwise, and where we used the independence of the components of $Y$ and the orthonormality of matrix $\mathcal{A}$. According to proposition 2, $X$ approaches a Gaussian vector as $N \rightarrow \infty$; its autocorrelation matrix is diagonal with elements $P$; therefore the components of $X$ are statistically independent, Gaussian distributed with zero mean and variance $P$.

The benefit of Gaussian-distributed source alphabet for various communication channels is a well-studied information theoretic topic.

\section{Diversity transform construction and optimization}

Diversity transform matrices for DST codes can be optimized based on maximizing the mutual information $I(R, Y)$ between $R$ and $Y$. Maximization of $I(R, Y)$, however, turns out to be a complex task [11]. Alternatively, we shall employ the cutoff rate as a measure with respect to which the diversity transform is optimized. The channel cutoff rate $R_{0}$ is a lower bound on the Shannon channel capacity $C$. Its usage in place of capacity often leads to tractable results. In this context, the detection is assumed to be maximum likelihood.

\subsection{Cutoff rate analysis}

Viewing an $N$-dimensional vector $Y$ as a 'super' symbol of an infinite-length (random) code in which all super symbols are statistically independent, the cutoff rate $R_{0}(N)$ for single channel use is given by [12]:

$$
2^{-R_{0}(N)}=E_{H}\left\{\int_{R}\left[\frac{1}{M^{N}} \sum_{Y} \sqrt{f_{R}(R / Y, H)}\right]^{2} d R\right\},
$$



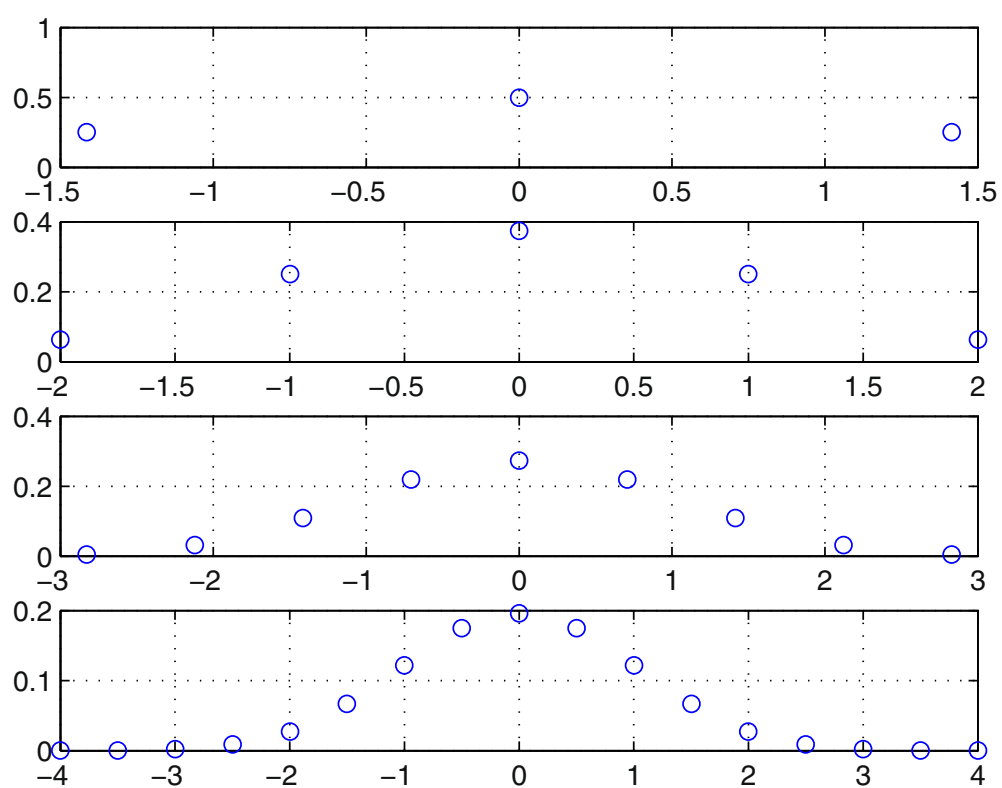

Figure 1 Alphabet distribution for increasing order transform: $H_{2}, H_{4}, H_{8}$ and $H_{16}$.

where $E_{H}$ denotes expectation over $H$.

When the path gains are known at the receiver side, the probability density function of $R$ conditioned on $Y$ and $H$ can be written as

$$
\begin{aligned}
f_{R}(R \mid Y, H)= & \frac{1}{\pi^{N} \cdot \operatorname{det}\left(\sigma^{2} \cdot H\right)} \exp \left\{-(R-H \mathcal{A} Y)^{\dagger}\right. \\
& \left.\cdot\left(\sigma^{2} H\right)^{-1} \cdot(R-H \mathcal{A} Y)\right\} .
\end{aligned}
$$

The cutoff rate $R_{0}$ for a single channel usage of each of the $N$ symbols constituting $Y$ is $R_{0}(N) / N$, thus combining Equations 11 and 12 one obtains

$$
\begin{gathered}
2^{-N R_{0}}=E_{H}\left\{\int _ { R } \left[\frac{1}{M^{N} \pi^{N / 2}\left(\sigma^{2}\right)^{N / 2} \sqrt{\operatorname{det}(H)}} .\right.\right. \\
\left.\left.\sum_{Y} \exp \left\{-\frac{1}{2}(R-H \mathcal{A} Y)^{\dagger} \cdot\left(\sigma^{2} H\right)^{-1} \cdot(R-H \mathcal{A} Y)\right\}\right]^{2} d R\right\} .
\end{gathered}
$$

For notational brevity, we introduce $Z$ as a dummy variable (of the same nature as $Y$ in Equation 13) and define $R_{Y} \equiv R-H \mathcal{A} Y$ and $R_{Z} \equiv R-H \mathcal{A} Z$; thus, we have

$$
\begin{aligned}
2^{-N R_{0}}= & E_{H}\left\{\int_{R} \frac{1}{M^{2 N \pi^{N}}\left(\sigma^{2}\right)^{N} \operatorname{det}(H)}\right. \\
& \times \sum_{Y} \sum_{Z} \exp \left[-\frac{1}{2}\left(R_{Y}\right)^{\dagger} \cdot\left(\sigma^{2} H\right)^{-1} \cdot R_{Y}\right] \\
& \left.\cdot \exp \left[-\frac{1}{2}\left(R_{Z}\right)^{\dagger} \cdot\left(\sigma^{2} H\right)^{-1} \cdot R_{Z}\right] d R\right\} \\
= & E_{H}\left\{\frac{1}{M^{2 N}} \sum_{Y} \sum_{Z} \prod_{j=1}^{N} I_{j}\right\}
\end{aligned}
$$

where $I_{j}$ is defined as

$$
\begin{aligned}
I_{j} & =\int_{r_{j}} \frac{1}{\pi \sigma^{2} \tilde{h}^{j}} \\
& \exp \left\{-\frac{\left|r_{j}-\tilde{h}^{j} \sum_{i=1}^{N} a_{j i} Y_{i}\right|^{2}+\left|r_{j}-\tilde{h}^{j} \sum_{i=1}^{N} a_{j i} Z_{i}\right|^{2}}{2 \sigma^{2} \tilde{h}^{j}}\right\} d r_{j} .
\end{aligned}
$$

Rearranging the algebraic terms in the last expression, we get

$$
\begin{aligned}
I_{j} & =\int_{r_{j}} \frac{1}{\pi \sigma^{2} \tilde{h}^{j}} \exp \left\{-\frac{\left|r_{j}-\frac{1}{2} \tilde{h}^{j} \sum_{i=1}^{N} a_{j i}\left(Y_{i}+Z_{i}\right)\right|^{2}}{\sigma^{2} \tilde{h}^{j}}\right\} d r_{j} . \\
\exp \left\{-\frac{\left(\tilde{h}^{j}\right)^{2}\left|\sum_{i=1}^{N} a_{j i}\left(Y_{i}-Z_{i}\right)\right|^{2}}{4 \sigma^{2} \tilde{h}^{j}}\right\} & \exp \left\{-\frac{\left(\tilde{h}^{j}\right)^{2}\left|\sum_{i=1}^{N} a_{j i}\left(Y_{i}-Z_{i}\right)\right|^{2}}{4 \sigma^{2} \tilde{h}^{j}}\right\}=\exp \left\{-\frac{\tilde{h}^{j} G_{j}}{\sigma^{2}}\right\},
\end{aligned}
$$


where

$$
G_{j}=\frac{\left|\sum_{i=1}^{N} a_{j i}\left(Y_{i}-Z_{i}\right)\right|^{2}}{4} .
$$

According to the channel model used, $\tilde{h}^{j}$ are statistically independent among different blocks; Equation 14 then becomes

$$
2^{-N R_{0}}=\frac{1}{M^{2 N}} \sum_{Y} \sum_{Z} \prod_{j=1}^{N} E_{\tilde{h}^{j}}\left\{I_{j}\right\} .
$$

The following proposition will be useful for the calculation of the expectation in Equation 17:

Proposition 4. $\tilde{h}^{j}$ is chi-square distributed with $2 m n$ degrees of freedom, where $n$ and $m$ are the number of transmit and receive antennas, respectively.

Proof.

$$
\tilde{h}^{t}=\sum_{i=1}^{n} \sum_{j=1}^{m}\left|h_{i, j}^{t}\right|^{2}=\sum_{i=1}^{n} \sum_{j=1}^{m} \Re\left(h_{i, j}^{t}\right)^{2}+\Im\left(h_{i, j}^{t}\right)^{2},
$$

which is the sum of the squares of $2 m n$ independent, $N(0,0.5)$-distributed, random variables. The distribution of $\tilde{h}^{t}$ is therefore

$$
f_{\tilde{h}^{t}}\left(\tilde{h}^{t}\right)=\frac{1}{(m n-1) !} \cdot\left(\tilde{h}^{t}\right)^{m n-1} e^{-\tilde{h}^{t}} U\left(\tilde{h}^{t}\right),
$$

with $U($.$) being the unit step function.$

Combining Proposition 4 with Equation 15 gives

$$
\begin{aligned}
E_{\tilde{h}^{j}}\left\{I_{j}\right\}= & \int_{0}^{\infty} \frac{1}{(m n-1) !} \cdot\left(\tilde{h}^{j}\right)^{m n-1} \\
& \cdot \exp \left[-\tilde{h}^{j}\left(1+\frac{G_{j}}{\sigma^{2}}\right)\right] d \tilde{h}^{t} \\
= & \frac{1}{\left(1+\frac{G_{j}}{\sigma^{2}}\right)^{m n}} .
\end{aligned}
$$

From Equations 17 and 18, it follows that

$$
R_{0}=\log _{2} M-\frac{1}{N} \log _{2}\left[\frac{1}{M^{N}} \sum_{Y} \sum_{Z} \prod_{j=1}^{N} \frac{1}{\left(1+\frac{G_{j}}{\sigma^{2}}\right)^{m n}}\right],
$$

where $G_{j}$ is defined in Equation 16. Note that the actual cutoff rate of a scheme which uses an orthogonal design with parameters $p, k$ is multiplied by a factor of $k / p$, since $p$ time slots are used to transmit $k$ symbols; the actual cutoff rate is therefore

$$
\begin{aligned}
& R_{0}(p, k) \\
& =\frac{k}{p}\left\{\log _{2} M-\frac{1}{N} \log _{2}\left[\frac{1}{M^{N}} \sum_{Y} \sum_{Z} \prod_{j=1}^{N} \frac{1}{\left(1+\frac{G_{j}}{\sigma^{2}}\right)^{m n}}\right]\right\} .
\end{aligned}
$$

It is evident from Equation 20 that in order to maximize $R_{0}$, one has to minimize the term

$$
F(\mathcal{A})=\sum_{Y} \sum_{Z} \prod_{j=1}^{N} \frac{1}{\left(1+\frac{G_{j}}{\sigma^{2}}\right)^{m n}} .
$$

This, in turn, is achieved by identifying 'good' unitary matrices $\mathcal{A}$ using the relation (16), between $G_{j}$ and $\mathcal{A}$, such that Equation 21 is minimized. This is the subject of the next subsections.

\subsection{Using gradient descent algorithm}

Unfortunately, matrices that minimize Equation 21, and hence optimize the cutoff rate, do not admit a closed form solution. In this subsection, we propose a gradient descent-based algorithm for finding a matrix, $\mathcal{A}$, that minimizes Equation $21 . \mathcal{A}$ is typically required to obey the following constraint

$$
\sum_{i, j=1}^{n}\left|a_{i j}\right|^{2}=n,
$$

so that the total average transmitted power remains constant. First, denote the complex derivative of the real function $F(\mathcal{A})$ with respect to $a_{k l}$ (the term in row $k$ and column $l$ of $\mathcal{A})$ as

$$
\frac{\Delta F(\mathcal{A})}{\triangle a_{k l}}=\frac{\partial F(\mathcal{A})}{\partial \Re\left(a_{k l}\right)}+i \frac{\partial F(\mathcal{A})}{\partial \Im\left(a_{k l}\right)} .
$$

A single iteration of gradient descent for minimizing Equation 21 is given by

$$
a_{k l}^{(i+1)}=a_{k l}^{(i)}-\left.\delta \cdot \frac{\Delta F(\mathcal{A})}{\triangle a_{k l}}\right|_{\mathcal{A}=\mathcal{A}^{(i)}},
$$

i.e., each iteration attempts to update $\mathcal{A}$ in a direction that makes $F(\mathcal{A})$ smaller. $\delta$ is a positive constant which determines the step size in each iteration. The derivative (23) can be calculated from Equation 21 as:

$$
\begin{array}{r}
\frac{\Delta F(\mathcal{A})}{\Delta a_{k l}}=\sum_{Y} \sum_{Z} \prod_{j=1}^{N} \frac{1}{\left(1+\frac{G_{j}}{\sigma^{2}}\right)^{m n}} \cdot(-m n) \\
\frac{1}{2 \sigma^{2}\left(1+\frac{G_{k}}{\sigma^{2}}\right)} \cdot\left[\left(Y_{l}-Z_{l}\right)^{*} \cdot \sum_{i=1}^{N} a_{k i}\left(Y_{i}-Z_{i}\right)\right],
\end{array}
$$


where each iteration of Equation 24 is followed by normalization of the matrix $\mathcal{A}$ according to Equation 22; this normalization is less acute for convergence of the algorithm when the step size $\delta$ gets smaller.

\subsection{Construction of DRT matrices using elementary unitary matrices}

An alternative approach for constructing DRT matrices is briefly described herein.

A unitary matrix with determinant equal to one can be constructed as the product of elementary unitary matrices with determinant equal to one [13]. An elementary unitary matrix is of the form

$$
T_{i j}=\left[\begin{array}{ccccccc}
1 & 0 & 0 & \ldots & \ldots & \ldots & 0 \\
0 & 1 & 0 & \ldots & \ldots & \ldots & 0 \\
\ldots & \ldots & \ldots & \ldots & \ldots & \ldots & \ldots \\
0 & \ldots & e^{j \varphi_{i j}} \cos \left(\phi_{i j}\right) & \ldots & -e^{j \theta_{i j}} \sin \left(\phi_{i j}\right) & \ldots & 0 \\
\ldots & \ldots & \ldots & \ldots & \ldots & \ldots & \ldots \\
0 & \ldots & e^{-j \theta_{i j}} \sin \left(\phi_{i j}\right) & \ldots & e^{-j \varphi_{i j}} \cos \left(\phi_{i j}\right) & \ldots & 0 \\
0 & \ldots & \ldots & \ldots & 0 & 1 & 0 \\
0 & \ldots & \ldots & \ldots & 0 & 0 & 1
\end{array}\right] .
$$

The elementary unitary matrix $T_{i j}$ has 3 degrees of freedom, and differs from the unit matrix in only four elements, located at the intersection of exactly two rows $i$ and $j$ with two columns $i$ and $j$ where $i<j$. We construct unitary matrices with determinant one as a product of $\frac{1}{2} N(N-1)$ elementary $N$-dimensional unitary matrices

$$
\mathcal{A}=\prod_{i=1}^{N} \prod_{j=i+1}^{N} T_{i j} .
$$

\subsection{Transform optimization - results}

The diversity transform can be optimized with respect to the cutoff rate using either one of the aforementioned methods. It follows from Equation 20 that the optimum diversity matrix, denoted $\mathcal{A}_{M, N}(m, n)$, is SNR dependent, with $n$ and $m$ being the number of transmit and receive antennas, respectively, and where $M=2,4,8$, and 16 correspond to BPSK, QPSK, 8-PSK, and 16-QAM constellations, respectively. Good matrices have been obtained by employing numerical optimization based on either the gradient descent algorithm in the form of Equation 25, or by manipulating the three degrees of freedom of each constituent matrix $T_{i, j}$ of Equation 27. The main advantage of the former approach over the latter is its simplicity, particularly for large transform orders $N$. The main disadvantage of the former approach lies in its dependency on initial value for the matrix $\mathcal{A}$ and step size $\delta$. When implemented correctly, both methods provide very similar results in terms of maximizing the cutoff rate. The cutoff rates thus obtained are plotted in Figure 2 as a function of SNR, for some $M, N, m$, and $n$ values. For all the cases shown, we assumed one receive antenna (i.e., $m=1$ ), and the maximum achievable rate is $1 \mathrm{bit} / \mathrm{sec} / \mathrm{Hz}$, where for $n=2$, we used the orthogonal code $G_{2}$ and BPSK modulation, and for $n=3$, we used the orthogonal code $G_{3}$ with QPSK modulation since the rate of $G_{3}$ is one half. Nice improvement in cutoff rate is observed with a transformation order of $N=3$ compared to the uncoded scheme (corresponding to $N=1$ ). An example of an optimal matrix $\mathcal{A}$ is given below, calculated using gradient descent algorithm as described in Subsection 4.2. It was derived for a scenario with $n=3$ transmit antennas, transformation order of $N=3$, rate $1 \mathrm{bit} / \mathrm{sec} / \mathrm{Hz}$ and SNR of $4 \mathrm{~dB}$. The parameters of the gradient descent algorithm were chosen as follows : initial step size, used in Equation 22, is $\delta=0.03$; the step size is decreased by a factor of 0.97 with each iteration. The initial value for the matrix $\mathcal{A}$ is a matrix of equal entries (normalized by a scalar to satisfy the condition (22)). After 100 iterations, the following matrix was derived:

$$
\begin{aligned}
& \mathcal{A}_{4,3}(1,3) \\
& =\left[\begin{array}{lll}
0.4943+0.2753 i & 0.1235-0.5696 i & 0.5631+0.1511 i \\
0.0976-0.5724 i & 0.3038+0.4693 i & 0.5218+0.2797 i \\
0.4785+0.3373 i & 0.3685+0.4604 i & -0.0484-0.5543 i
\end{array}\right] .
\end{aligned}
$$

Employing this matrix in deriving $\left\{G_{j}\right\}_{j=1}^{3}$ via Equation 16 followed by substitution into Equation 20, a cutoff rate of $0.965 \mathrm{bit} / \mathrm{sec} / \mathrm{Hz}$ is achieved, compared to an uncoded scheme (where $\mathcal{A}$ is taken to be the identity matrix of size 3) whose cutoff rate is $0.92 \mathrm{bit} / \mathrm{sec} / \mathrm{Hz}$.

\section{Detection of DST codes}

We first describe an optimum detection algorithm for DST codes. Since optimum detection can be quite computationally complex, we also derive a linear detector for these codes. For linear detection, we shall introduce and justify the use of DRT matrices different from those obtained in the previous section.

\subsection{Optimum detection}

Optimum detection of DST codes, in the sense of minimizing the bit error probability, can be carried out in two steps:

1. Decode the orthogonal ST code by employing Proposition 1.

2. Reconstruct the source bits using maximum a posteriori (MAP) criterion according to the specific diversity matrix $\mathcal{A}$ applied.

Section 6 provides simulation results using this detection approach. Performing maximum a posteriori detection, as described above, is quite computationally involved even when using low order diversity transforms. Hence, a 


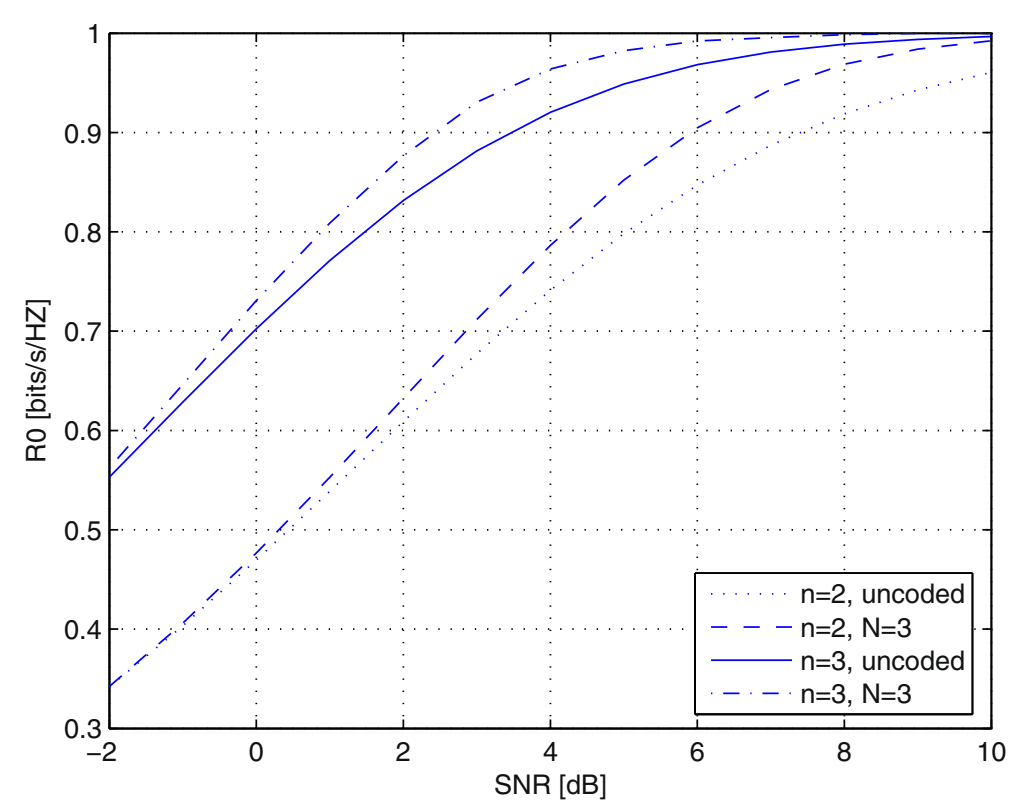

Figure 2 Optimal cuttoff rates, R0, for different transform orders and antenna array sizes.

simpler, suboptimum detection method is proposed in the form of linear detection.

\subsection{Linear detection - the MMSE receiver}

The detection rule is of the form

$$
Y^{\prime}=B R+h,
$$

where $R$ is given by Equation 9. The matrix $B$ and the vector $h$ are chosen to minimize the mean square error (MSE) of the receiver:

$$
\mathrm{MSE}=E\left\{\epsilon \epsilon^{\dagger}\right\}=E\left\{\left(Y-Y^{\prime}\right) \cdot\left(Y-Y^{\prime}\right)^{\dagger}\right\} .
$$

Assuming, as before, that $H$ (defined in Equation 10) is known to the receiver, the components of $Y$ are independent and identically distributed (i.i.d) with zero mean and energy $E_{s}$, the MMSE (minimum MSE) receiver is given by

$$
Y^{\prime}=E_{s} \cdot(H \mathcal{A})^{\dagger}\left[E_{s} \cdot(H \mathcal{A}) \cdot(H \mathcal{A})^{\dagger}+\sigma^{2} \cdot H\right]^{-1} \cdot R .
$$

Owing to the orthonormality of $\mathcal{A}$, the MMSE receiver can be formulated simply as:

$$
Y^{\prime}=\mathcal{A}^{\dagger} \Lambda R
$$

where $\Lambda$ is a diagonal matrix whose non-zero elements are as follows:

$$
\lambda_{i i}=\frac{E_{s} \cdot \tilde{h}^{i}}{E_{s} \cdot\left(\tilde{h}^{i}\right)^{2}+\sigma^{2} \cdot \tilde{h}^{i}} .
$$

Substituting Equation 9 into Equation 28, we get

$Y^{\prime}=\mathcal{A}^{\dagger} \Lambda(H \mathcal{A} Y+\Gamma)=\mathcal{A}^{\dagger} \Lambda H \mathcal{A} Y+\mathcal{A}^{\dagger} \Lambda \Gamma=\mathcal{A}^{\dagger} P \mathcal{A} Y+\mathcal{A}^{\dagger} \Lambda \Gamma$, where $P=\Lambda H$ is a diagonal matrix with elements given by

$$
p_{i i}=\frac{E_{s} \cdot\left(\tilde{h}^{i}\right)^{2}}{E_{s} \cdot\left(\tilde{h}^{i}\right)^{2}+\sigma^{2} \cdot \tilde{h}^{i}} ;
$$

the $j$ th element of $Y^{\prime}$ is given by

$$
y_{j}^{\prime}=y_{j} \sum_{i=1}^{N}\left|a_{i j}\right|^{2} p_{i i}+\sum_{k=1, k \neq j}^{N} \sum_{i=1}^{N} p_{i i} a_{i j}^{*} a_{i k} y_{k}+\sum_{i=1}^{N} g_{i i} a_{i j}^{*} \gamma_{i} .
$$

From Equation 29, it follows that the MMSE receiver is biased. This bias can degrade BER performance for large signal constellations. In order to eliminate this biasing, each element $y_{j}^{\prime}$ is divided by $\sum_{i=1}^{N}\left|a_{i j}\right|^{2} p_{i i}$.

It can be shown [5] that in order to minimize the variance of the MSE (when represented as a function of the channel fadings) the non-zero elements of the matrix $\mathcal{A}$ should satisfy

$$
\left|a_{i j}\right|=\frac{1}{\sqrt{N}}
$$

we shall employ the normalized Hadamard matrix, which satisfies this property.

\subsection{Suboptimum detection}

In view of the previous subsection, suboptimum detection can be summarized as follows:

1. Decode the orthogonal ST code by employing Proposition 1. 


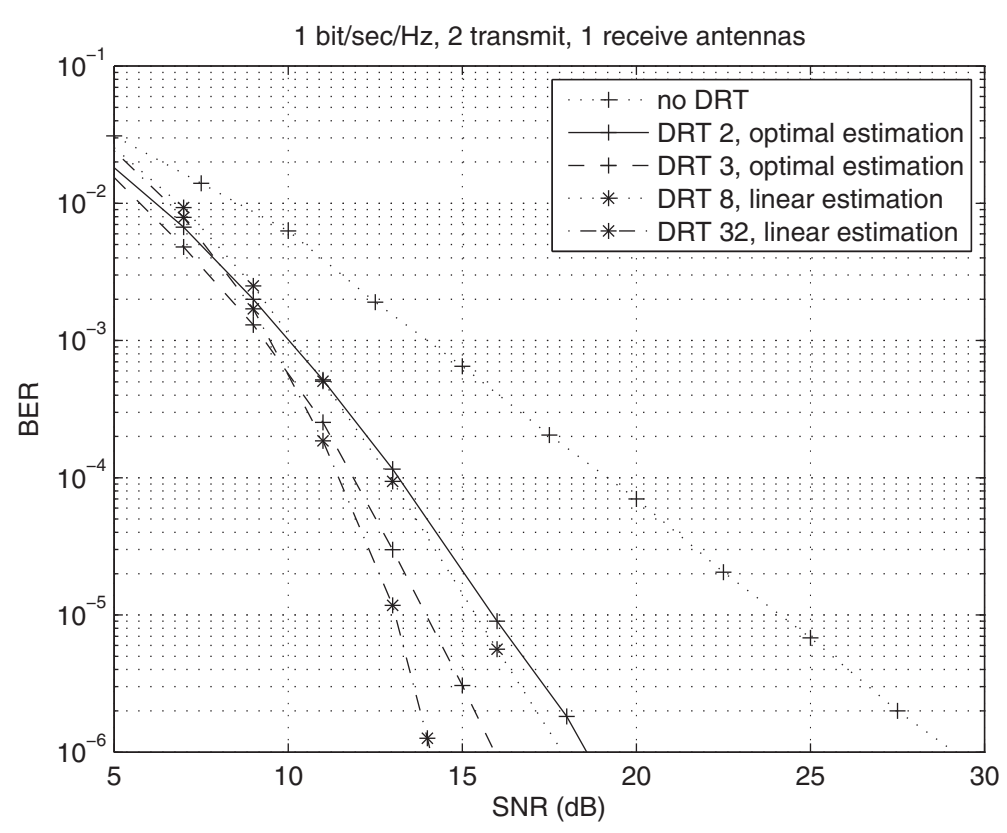

Figure 3 Bit error rate versus SNR for codes at $1 \mathrm{bit} / \mathrm{s} / \mathrm{Hz}$; two transmit, one receive antenna.

2. Use the linear MMSE receiver described in this section to get an estimate of $Y$. Recover the corresponding source bits.

The performance of the proposed DST codes and detection algorithms are quantified in the next section by computer simulations.

\section{Simulation results}

This section provides bit error rate performance obtained via simulations for various cases ${ }^{\mathrm{b}}$. Figures 3,4 , and 5 present bit error rate performance for transmission of $1 \mathrm{bit} / \mathrm{s} / \mathrm{Hz}$ using two to four transmit antennas, one receive antenna, DRT order $N$ of 1 to 3 when employing MAP decoding (note that $N=1$ amounts to employing

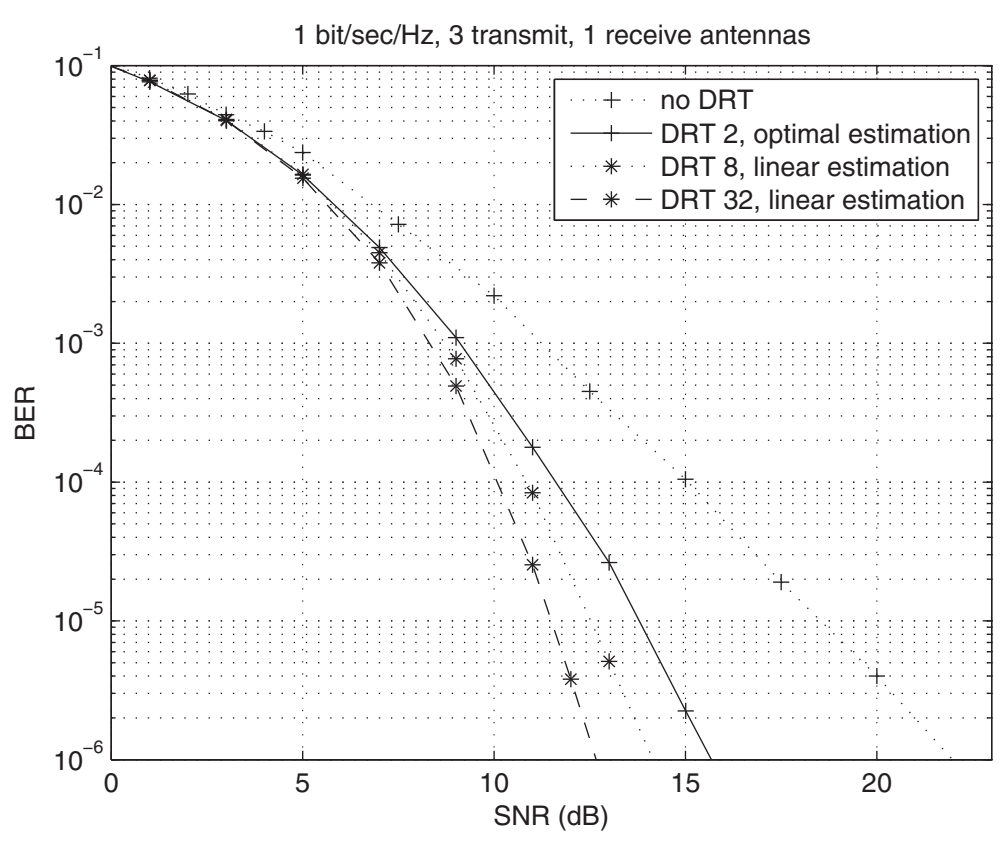

Figure 4 Bit error rate versus SNR for codes at $1 \mathrm{bit} / \mathrm{s} / \mathrm{Hz}$; three transmit, one receive antenna. 


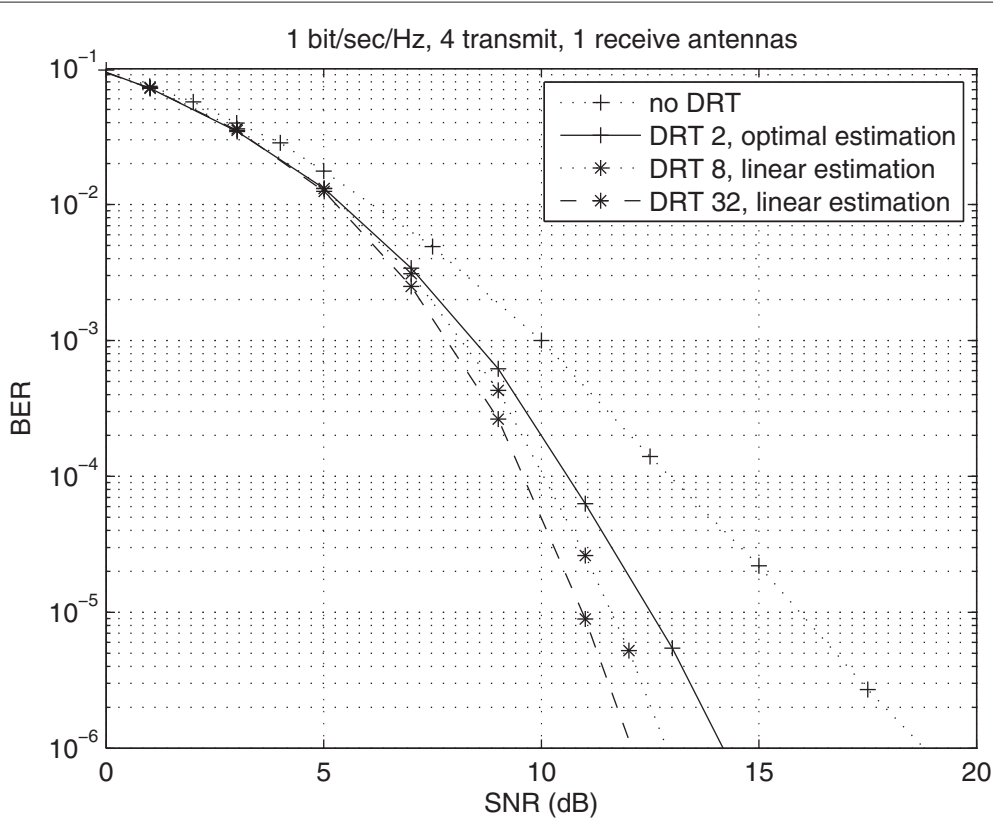

Figure 5 Bit error rate versus SNR for codes at $1 \mathrm{bit} / \mathrm{s} / \mathrm{Hz}$; four transmit, one receive antenna.

standard ST coding with no DRT). For high DRT orders of $N=8$ and $N=32$, also shown in the figures, we employ a suboptimal, yet reduced complexity, linear detection.

For the two-transmit antenna case, BPSK has been used with the code $G_{2}$. For three and four transmit antennas, we used QPSK with codes $G_{3}$, and $G_{4}$, respectively. It can be seen, for bit error rate $10^{-6}$, that order-2 DRT provides about 10,6 , and $5 \mathrm{~dB}$ coding gain for two, three, and four transmit antennas, respectively, as compared to regular (non-diversified) ST code. The corresponding gains for orders 8 and 32 are 11,8 , and $6 \mathrm{~dB}$ and 15, 9.5, and $7 \mathrm{~dB}$, respectively.

Note that the gain decreases as the number of transmit antennas increases. This can be attributed to the fact that a large number of antennas already exploit much of the attainable diversity gain. For the same reason, when the

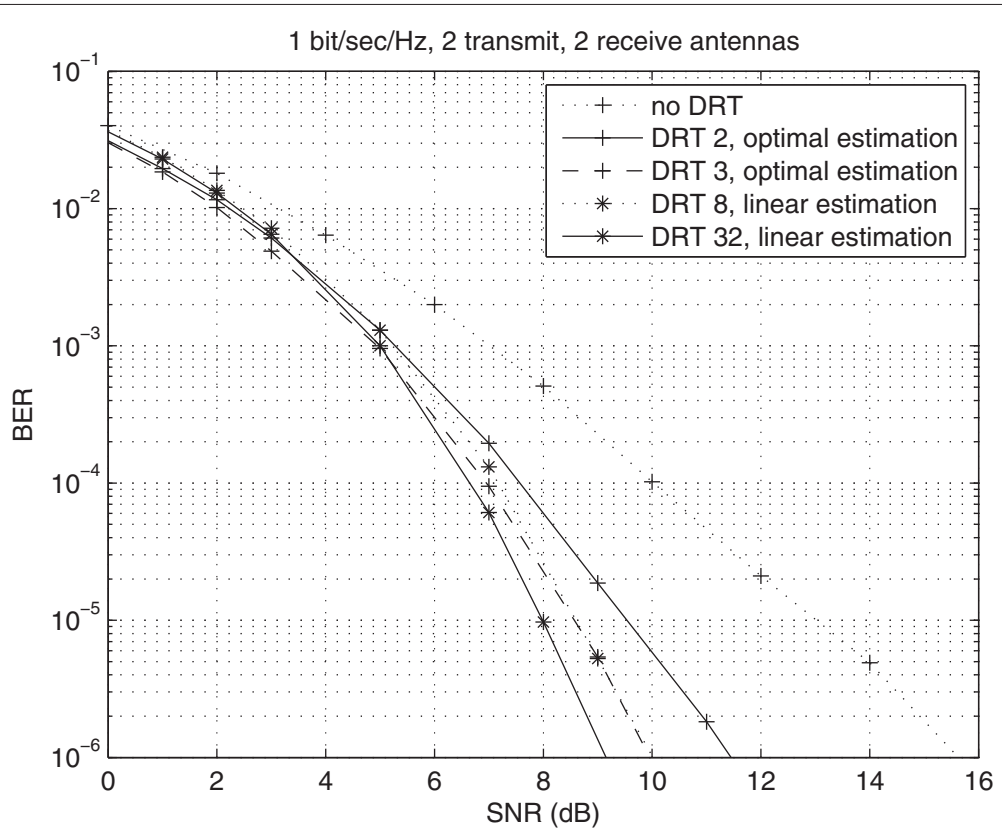

Figure 6 Bit error rate versus SNR for codes at $1 \mathrm{bit} / \mathrm{s} / \mathrm{Hz}$; two transmit, two receive antenna. 


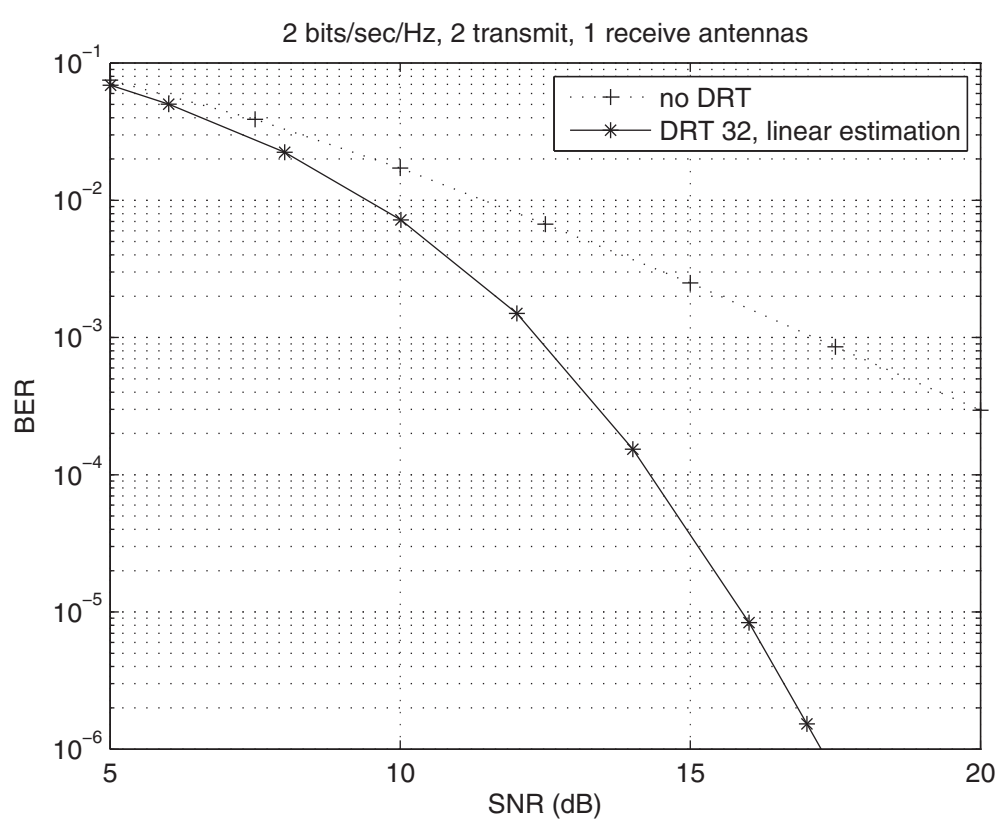

Figure 7 Bit error rate versus SNR for codes at $2 \mathrm{bits} / \mathrm{s} / \mathrm{Hz}$; two transmit, one receive antenna.

number of receive antennas is increased to two (Figure 6), the obtained gains are 4,2 , and $1.5 \mathrm{~dB}$ for two, three, and four transmit antennas $(N=2)$, respectively; 5.5 , 3.5 , and $2.5 \mathrm{~dB}(N=8$ with linear detection); and 6.5, 4, and $2.7 \mathrm{~dB}(N=32$ with linear detection), respectively. Clearly, in all scenarios where linear detection is involved, larger gains are obtained (with reduced complexity) due to employing increased DRT order; this is achieved for the price of somewhat increased latency at the receiver.

Figures 7 and 8 show bit error probability results for transmission of 2 bits $/ \mathrm{s} / \mathrm{Hz}$ with two to three transmit antennas, one receive antenna, and diversity order 1 and 32. The transmission using two antennas employs QPSK constellation with the code $G_{2}$. For three transmit

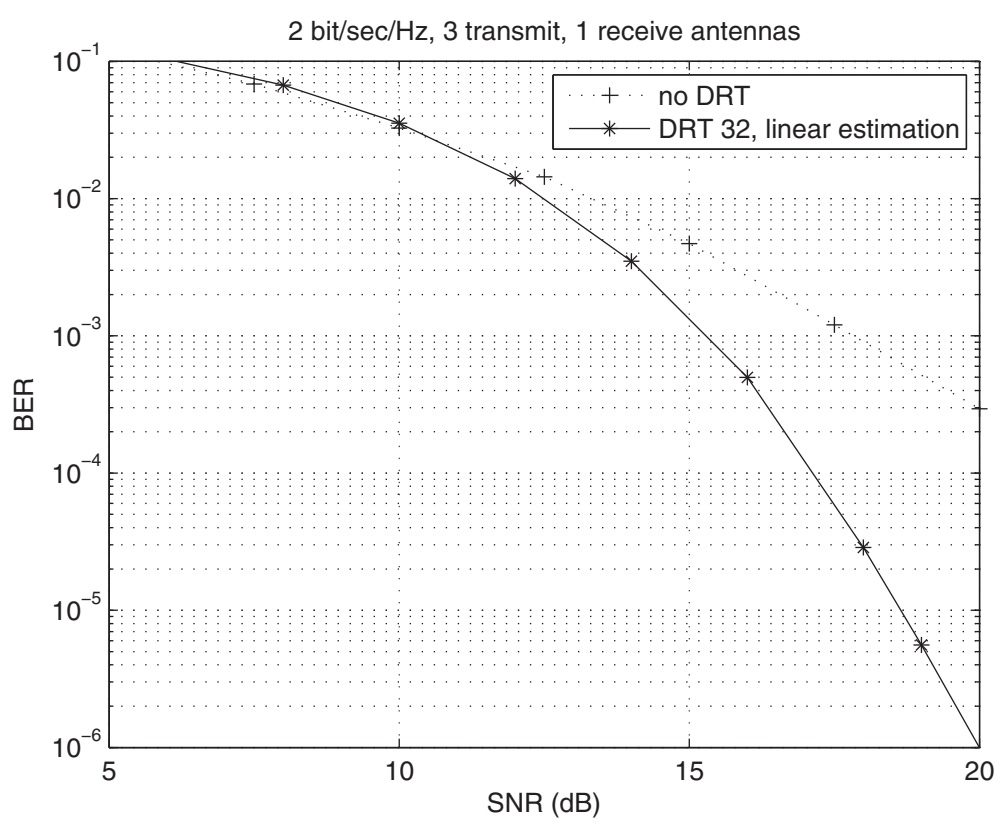

Figure 8 Bit error rate versus SNR for codes at $2 \mathrm{bits} / \mathrm{s} / \mathrm{Hz}$; three transmit, one receive antenna. 
antennas, 16-QAM constellation is used with the code $G_{3}$.

Extensive simulations were carried out also for higher spectral efficiencies. Consider transmission of $3 \mathrm{bits} / \mathrm{s} / \mathrm{Hz}$ using two to four transmit antennas and one receive antenna. For two transmit antennas, 8-PSK and the code $G_{2}$ have been used. For three and four transmit antennas, we used 16-QAM along with the codes $H_{3}$ and $H_{4}$, respectively. For bit error rate of $10^{-6}$, diversity transform of order 2 provides about $9,5.2$, and $3 \mathrm{~dB}$ gain for two, three, and four transmit antennas, respectively. Diversity transforms of order 8 and 32 provide gains of 6,5 , and 4.2 $\mathrm{dB}$ and $12.5,8$, and $5.2 \mathrm{~dB}$, respectively, when using linear detection.

Finally, it is demonstrated, for the block-fading channel, that when a communication scheme is limited by the number of antennas, the diversity transform can effectively compensate for the loss in spatial diversity. An upper bound on the error probability of codes using an orthogonal design is given by [14]

$$
P_{\text {error }} \doteq S N R^{-m n(1-r)}
$$

where $r$ is the spatial multiplexing gain and $\doteq$ denotes exponential equality.

Assume given a coding scheme employing $n$ transmit antennas, $m$ receive antennas and diversity transform of order $N$ as proposed herein. It is interesting to compare this scheme with a scheme having $n$ transmit and $N \cdot m$ receive antennas.

In view of the error exponent (30), the latter scheme can be used to lower bound the performance of the proposed scheme. The latter scheme can achieve spatial diversity gain of $N \cdot m n$, while that of the first scheme depends on the temporal diversity gain provided by the diversity transform. Comparing Figures 3, 4, and 5 (for $N=2$ ) with Figure 6 and the results for three to four transmit, two receive antennas (with no DRT) reveal that the latter curves are about 2 to $3 \mathrm{~dB}$ better than the former set of curves. This is due to the fact that a short transform cannot achieve the full diversity provided by a large antenna array. However, when a communication scheme is limited by the number of antennas, the diversity transform can effectively compensate for this loss in spatial diversity.

\section{Conclusions}

Space-time block codes of increased diversity are constructed for MIMO Rayleigh fading channels. This construction combines orthogonal designs with diversity transforms, thus providing ST block coding scheme of high diversity gain that can be easily tailored for any antenna array and signal constellation. Two detection algorithms are described: optimum detection and suboptimum detection employing linear estimation at the receiver. Specific construction of codes with bandwidth efficiencies of 1,2 , and 3 (bits/s/ $\mathrm{Hz}$ ) are detailed. Among the properties of DST codes, we can mention its high diversity, low implementation complexity, high transmission rates, and the fact that the distribution of the obtained code alphabet is close to Gaussian. Finally, we note that quasi-orthogonal codes $[15,16]$ can be used in the framework of DST codes in order to achieve even higher data rates.

\section{Endnotes}

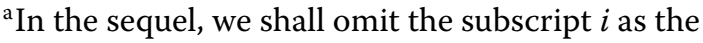
analysis is the same for any value $i$.

${ }^{\mathrm{b}}$ Hereinafter, whenever the detector is linear, we employed the Hadamard transform of appropriate dimensions as the DRT matrix. Otherwise, we employed the optimal matrices obtained in Section 4.

\section{Appendix}

\section{ST codes used in this work}

Rate $\frac{1}{2}$ ST orthogonal codes are given by:

$$
G_{3}=\left(\begin{array}{ccc}
x_{1} & x_{2} & x_{3} \\
-x_{2} & x_{1} & -x_{4} \\
-x_{3} & x_{4} & x_{1} \\
-x_{4} & -x_{3} & x_{2} \\
x_{1}^{*} & x_{2}^{*} & x_{3}^{*} \\
-x_{2}^{*} & x_{1}^{*} & -x_{4}^{*} \\
-x_{3}^{*} & x_{4}^{*} & x_{1}^{*} \\
-x_{4}^{*} & -x_{3}^{*} & x_{2}^{*}
\end{array}\right) \quad G_{4}=\left(\begin{array}{cccc}
x_{1} & x_{2} & x_{3} & x_{4} \\
-x_{2} & x_{1} & -x_{4} & x_{3} \\
-x_{3} & x_{4} & x_{1} & -x_{2} \\
-x_{4} & -x_{3} & x_{2} & x_{1} \\
x_{1}^{*} & x_{2}^{*} & x_{3}^{*} & x_{4}^{*} \\
-x_{2}^{*} & x_{1}^{*} & -x_{4}^{*} & x_{3}^{*} \\
-x_{3}^{*} & x_{4}^{*} & x_{1}^{*} & -x_{2}^{*} \\
-x_{4}^{*} & -x_{3}^{*} & x_{2}^{*} & x_{1}^{*}
\end{array}\right) ;
$$

and rate $\frac{3}{4}$ ST orthogonal codes given by:

$$
\begin{aligned}
& H_{3}=\left(\begin{array}{ccc}
x_{1} & x_{2} & \frac{x_{3}}{\sqrt{2}} \\
-x_{2}^{*} & x_{1}^{*} & \frac{x_{3}}{\sqrt{2}} \\
\frac{x_{3}^{*}}{\sqrt{2}} & \frac{x_{3}^{*}}{\sqrt{2}} & \frac{\left(-x_{1}-x_{1}^{*}+x_{2}-x_{2}^{*}\right)}{2} \\
\frac{x_{3}^{*}}{\sqrt{2}} & -\frac{x_{3}^{*}}{\sqrt{2}} & \frac{\left(x_{2}+x_{2}^{*}+x_{1}-x_{1}^{*}\right)}{2}
\end{array}\right) \\
& H_{4}=\left(\begin{array}{cccc}
x_{1} & x_{2} & \frac{x_{3}}{\sqrt{2}} & \frac{x_{3}}{\sqrt{2}} \\
-x_{2}^{*} & x_{1}^{*} & \frac{x_{3}}{\sqrt{2}} & -\frac{x_{3}}{\sqrt{2}} \\
\frac{x_{3}^{*}}{\sqrt{2}} & \frac{x_{3}^{*}}{\sqrt{2}} & \frac{\left(-x_{1}-x_{1}^{1}+x_{2}-x_{2}^{*}\right)}{2} & \frac{\left(-x_{2}-x_{2}^{*}+x_{1}-x_{1}^{*}\right)}{2} \\
\frac{x_{3}^{*}}{\sqrt{2}} & -\frac{x_{3}^{*}}{\sqrt{2}} & \frac{\left(x_{2}+x_{2}^{*}+x_{1}-x_{1}^{*}\right)}{2} & -\frac{\left(x_{1}+x_{1}^{*}+x_{2}-x_{2}^{*}\right)}{2}
\end{array}\right)
\end{aligned}
$$

Competing interests

The authors declare that they have no competing interests.

\section{Acknowledgements}

The authors wish to thank the anonymous reviewers for their comments and suggestions. 
Received: 29 May 2013 Accepted: 30 December 2013

Published: 15 January 2014

\section{References}

1. SM Alamouti, A simple transmit diversity technique for wireless communications. IEEE J. Sel. Areas in Commun. 16, 1451-1458 (1998)

2. VTarokh, H Jafarkhani, AR Calderbank, Space-time block codes from orthogonal designs. IEEE Trans. on Inform. Theory 45, 1456-1467 (1999)

3. X Giraud, E Boutillon, JC Belfiore, Algebraic tools to build modulation schemes for fading channels. IEEE Trans. on Inform. Theory. 43, 938-952 (1997)

4. E Bayer-Fluckiger, F Oggier, E Viterbo, New algebraic constructions of rotated $Z^{n}$-lattice constellations for the Rayleigh fading channel. IEEE Trans. on Inform. Theory 50, 702-714 (2004)

5. D Rainish, Diversity transform for fading channels. IEEE Trans. on Comm. 44, 1653-1661 (1996)

6. AJ Salomon, O Amrani, Space-time block codes using diversity transform, in Proc. IEEE Information Theory Workshop on Wireless Networks (Solstrand, 1-6 Jul 2007), pp. 1-5

7. HF Lu, Explicit constructions of multi-block space-time codes that achieve the diversity-multiplexing tradeoff, in Proc. IEEE International Symposium on Information Theory (Seattle, 9-14 July 2006), pp. 1149-1153

8. X Lin, RS Blum, Improved space-time codes using serial concatenation. IEEE Commun. Letters. 4, 221-223 (2000)

9. J Boutros, E Viterbo, Signal space diversity: a power- and bandwidth-efficient diversity technique for the Rayleigh fading channel. IEEE Trans. on Inform. Theory 44, 1453-1467 (1998)

10. AJ Salomon, O Amrani, Product construction of codes for Rayleigh-fading channels. IEEE Trans. on Inform. Theory 53, 416-428 (2007)

11. A Salomon, Iterated Code Constructions, (Tel Aviv University, Tel Aviv Israel, 2011)

12. N Abramson, Information Theory and Coding, (McGraw-Hill, New York, 1963)

13. DK Faddeev, VN Faddeeva, Computational Methods of Linear Algebra, (WH Freeman and Company, New York, 1963)

14. L Zheng, DNC Tse, Diversity and multiplexing: a fundamental tradeoff in multiple-antenna channels. IEEE Trans. on Inform. Theory 49, 1073-1096 (2003)

15. H Jafarkhani, A quasi-orthogonal space-time block code. IEEE Trans. Comm. 49, 1-4 (2001)

16. C Yuen, YL Guan, TT Tjhung, Quasi-orthogonal space-time block codes with minimum decoding complexity. IEEE Trans. Wireless Comm. 4, 2089-2094 (2005)

\section{Submit your manuscript to a SpringerOpen ${ }^{\circ}$ journal and benefit from:}

- Convenient online submission

- Rigorous peer review

- Immediate publication on acceptance

- Open access: articles freely available online

- High visibility within the field

- Retaining the copyright to your article

Submit your next manuscript at $>$ springeropen.com 